\title{
Access Network Selection Based on Fuzzy Logic and Genetic Algorithms
}

\author{
Mohammed Alkhawlani and Aladdin Ayesh \\ Faculty of Computing Sciences and Engineering, De Montfort University, Leicester LE1 9BH, UK \\ Correspondence should be addressed to Aladdin Ayesh, aayesh@dmu.ac.uk \\ Received 17 September 2007; Accepted 20 February 2008 \\ Recommended by Adel Alimi
}

In the next generation of heterogeneous wireless networks (HWNs), a large number of different radio access technologies (RATs) will be integrated into a common network. In this type of networks, selecting the most optimal and promising access network (AN) is an important consideration for overall networks stability, resource utilization, user satisfaction, and quality of service (QoS) provisioning. This paper proposes a general scheme to solve the access network selection (ANS) problem in the HWN. The proposed scheme has been used to present and design a general multicriteria software assistant (SA) that can consider the user, operator, and/or the QoS view points. Combined fuzzy logic (FL) and genetic algorithms (GAs) have been used to give the proposed scheme the required scalability, flexibility, and simplicity. The simulation results show that the proposed scheme and SA have better and more robust performance over the random-based selection.

Copyright ( 2008 M. Alkhawlani and A. Ayesh. This is an open access article distributed under the Creative Commons Attribution License, which permits unrestricted use, distribution, and reproduction in any medium, provided the original work is properly cited.

\section{Introduction}

The current radio resource management (RRM) [1-3] solutions and mechanisms for the wireless networks consider only the case of a single RAT where mobile users can only access that RAT and coexisting subnetworks can only be operated independently. Common RRM (CRRM) [4-7] and joint RRM (JRRM) [8-11] are two types of the processes that enable the management within a single and between the different RATs of the HWN. CRRM is a cooperative RRM architecture proposed by 3 GPP $[4,5]$ to make UMTS and GSM/GPRS networks cooperate. It is responsible for coordinating the individual RRM entities of each RAT. JRRM is another cooperative RRM architecture and mechanism proposed by IST SCOUT [10] and TRUST [11] projects and is similar to CRRM, but it is not restricted to GSM and UMTS and complements the CRRM with additional features and algorithms. Both JRRM and CRRM imply the use of some new RRM mechanisms such as access network selection (ANS), joint admission control (JAC), joint scheduling control (JSC), and vertical handover (VHO). Our work concentrates on the first mechanism, that is, ANS, which decides how to select the most suitable access network (AN) based on the discovered accesses, QoS constraints, operator policies, user preferences and available system capacity, and utilization.

Not many algorithms to the ANS problem can be encountered in the literature so far. In [12], there are different signal thresholds for each AN. Mobile node compares the RSS with the signal thresholds and decides vertical handoff procedure. In [13], mobile nodes keep connection with the higher throughput network until RSS goes below a threshold. In [14], service types are considered and differentiating service is achieved by different levels of RSS. A network selection algorithm that is based on the distinct features of voice and data traffic is proposed in [15]. In this algorithm, the new calls prefer the cellular network for voice service and WLANs for the data service. In general, the current conventional single criteria-based algorithms such as the proposed [12-14] are rigid and take only one criterion and cannot react easily to the changing environment conditions and accumulated human knowledge. Usually they cannot cope with the different view points and goals of the operators, users, and QoS requirements which make them inefficient for a multicriteria problem such as ANS problem. 


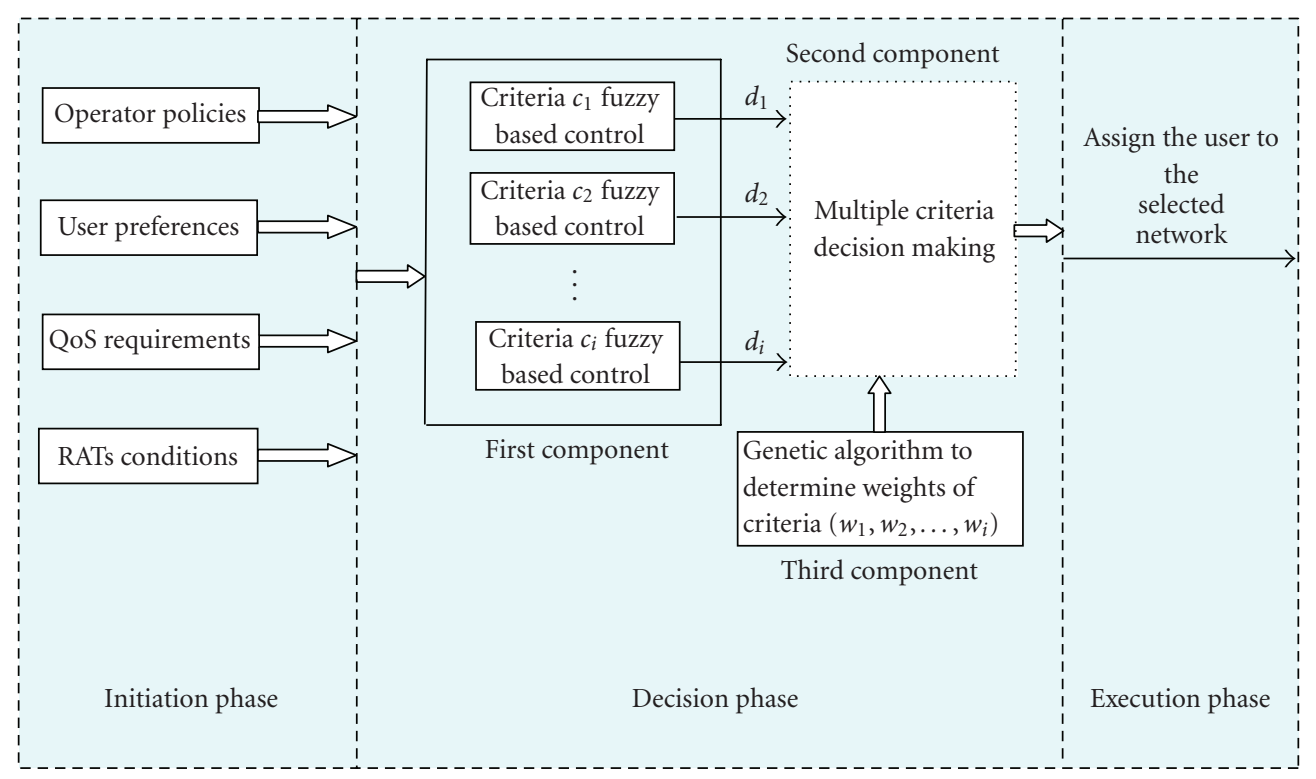

FIGURE 1: The proposed solving scheme for access network selection problem based on FL, MCDM, and GA.

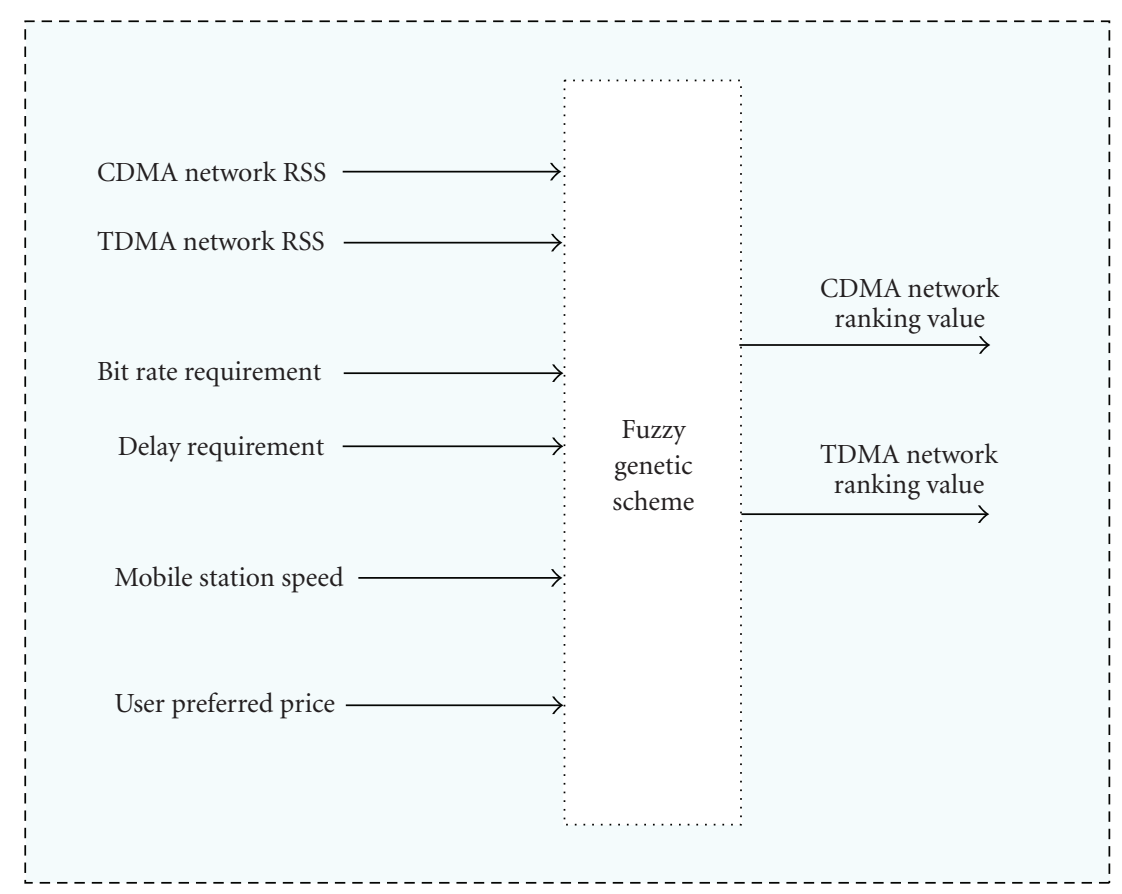

FIGURE 2: ANS SA for coexisted CDMA and TDMA systems.

Several policy-based RAT selection solutions are proposed in [16-18]. General frameworks for a policy-based RAT selection with some specific examples are proposed by Gelabert et al. [16]. A network capacity surfaces policybased admission controller is presented by Murray and Pesch [17]. A policy-based handover mechanism for mobile multihomed hosts is presented in [18] by Ylitalo et al. The handover decision is based on explicit user defined policies, and considers different criteria in the order of user defined priority. These solutions do not consider the tradeoff between criteria of the ANS problem and does not specify the importance and sensitivity of each criterion to the selection problem.

Several multicriteria decision making-based algorithms are proposed [19-23]. These conventional multicriteriabased algorithms do not take into account the complexities and uncertainties that arise from the different characteristics and natures of the different RATs. For these algorithms, it is not an easy task to incorporate the accumulated human knowledge about the problem and the only method to adapt 
the algorithms is to change the criteria weights randomly to get better results. In addition, these algorithms do not have a proper method to address the importance of the different criteria to the ANS.

Several multicriteria-based algorithms with the aid of artificial intelligence tools such as fuzzy logic, neural networks, and genetic algorithms [24-26] are suffering from scalability and modularity problems. They cannot cope easily with the increased numbers of RATs and criteria in the HWN. These algorithms take all inputs from the different RATs at once to one fuzzy logic block, so they suffer from scalability and complexity problems when more RATs or membership functions added due to the exponential increase on the number of inference rules.

It is worth to mention that most of the above algorithms do not addressed clearly the roles of the users and the operators in the selection process. Furthermore, some of the current algorithms could not differentiate between the initial selection process for the new service request and the selection process during the $\mathrm{VHO}$ process.

The main contribution of this paper is the development of a new class of ANS algorithms that is based on hybrid parallel fuzzy logic (FL)-based decision, multiple-criteria decision making (MCDM) systems, and genetic algorithms (GAs). This class of algorithms represents the first attempt to develop adaptive, flexible, and scalable ANS algorithms that are utilize the advantages of hybrid parallel FL decisionmaking systems and MCDM systems. Up to our knowledge, no utilization for GA or parallel FL-based decision abilities for ANS has been carried out so far.

Our proposed scheme to solve the ANS problem is described in Section 2. Software assistant (SA) based on the proposed scheme in coexisted CDMA- and TDMA-based systems is presented in Section 3. The simulation models and performance evaluation are carried out in Section 4. Conclusions are presented in Section 5.

\section{ANS Problem Solving Scheme}

More intelligent and robust solutions than the existed ones are needed. In general, any ANS solution has to take into its account the following general requirements.

(i) The solution has to solve the ANS problem in a simple way to give a reasonable and acceptable delay before the decision appears.

(ii) The solution has to cope with the different view points and goals of the operators and the users and to give both parties the right and fair role in the selection process.

(iii) The solution has to react to the changing environment conditions and accumulated human knowledge about the problem.

(iv) The solution has to allow any type of inputs and to be applicable to any type of ANs.

(v) The solution has to be able to handle the increasing number of RATs and it also has to be able to handle a large number of criteria.
The ANS procedure can be divided into three phases, initiation phase, decision phase, and execution phase. The objective of the initiation phase is to recognize the need for ANS and subsequently initiate it and find out the required information and measurements for the decision phase. In the decision phase, a comparison of the information and the measurements calculated from a variety of sources including networks measurements, QoS requirements, user preferences, and operator policies. This comparison lead to the identification of the best available AN according to the defined performance evaluation metrics. In the execution phase, the user is assigned to the selected network in the decision phase.

A generic scheme to solve the ANS problem and any other similar selection problem is shown in Figure 1. The scheme decision phase consists of three main components, the first component contains a set of small parallel fuzzy logic (FL)-based subsystems, the second component is a multiplecriteria decision making (MCDM) system, and the third component is a genetic algorithm (GA)-based component to assign a suitable weight for the criteria in the second component. The scheme decision phase can be described in more detail as follows.

(i) The heterogeneous wireless environment contains up to $n \mathrm{ANs}\left(\mathrm{RAT}_{1}, \mathrm{RAT}_{2}, \ldots, \mathrm{RAT}_{n}\right)$ and the framework has to select the most promising one or to rank the RATs according to their suitability.

(ii) The selection depends on multiple criteria up to $i\left(c_{1}, c_{2}, \ldots, c_{i}\right)$. Different type of criteria can be measured from different sources to cover the different view points of the users, the operators, the applications, and the network conditions. Each criterion is measured then passed to its FL-based control subsystem in the first component.

(iii) Every FL-based subsystem gives an initial score for each RAT that reflects the suitability of that RAT according the FL subsystem criterion. The different sets of scores $\left(d_{1}, d_{2}, \ldots, d_{i}\right)$ are sent to the MCDM in the second component.

(iv) The GA component assigns a suitable weight $\left(w_{1}, w_{2}, \ldots, w_{i}\right)$ for each initial decision according to the objective function that is specified by the operator according to the importance and sensitivities of ANS criteria to the different characteristics of a wireless heterogeneous environment.

(v) Using the initial scores coming from the first component and the weights that are assigned manually or using the third component, the MCDM will select the most promising AN or will rank the available RATs according to their suitability.

Our proposed algorithm utilizes the advantages of parallel FL control and MCDM, and GA. The following subsections summarize the main advantages and strengths of our proposed ANS. 


\subsection{The Advantages of the Application of FL to the ANS Problem}

The framework utilizes the advantages of the application of FL to the ANS problem that can be summarized as follows.

(i) Because of the HWN complex nature, any proposed ANS has to allow the solution of the ANS problem in a simple way and to avoid the complex analytical and mathematical equations used to in the conventional solutions. FL is conceptually easy to understand and easy to apply. The mathematical concepts behind fuzzy reasoning are very simple. The simplicity of the FL helps in providing the required simple ANS solution.

(ii) The data, information, and measurements that have to be taken into account in the ANS are in general very dissimilar, imprecise, contradictory, and coming from different sources. For example, some input parameters such as the received signal strength, the cell loads, and the SNR from different RATs are not directly comparable. FL is tolerant of imprecise data and it allows imprecise and contradictory inputs. As a result of that, an FL-based solution has been thought to be a good candidate for reaching suitable ANS decisions from such imprecise and dissimilar information.

(iii) FLC reconciles the conflicting objectives of the operators, users, and applications about the ANS.

(iv) ANS solution has to be able to response to the changing conditions of the HWN environments and the accumulated experience of the operators and users. FL-based solution is easy to modify by tuning and adjusting the inference rules and membership functions.

(v) The use of linguistic variables and inference rules make the FL-based solution similar to the way humans think, which makes it more intelligent and powerful than the conventional algorithm. This also can help in easy incorporation for the accumulated operator and user knowledge about the ANS problem.

\subsection{Advantages of Using Parallel FLC to the ANS Problem}

Applying the parallel FLC rather than the traditional FLC has some advantages when solving the ANS problem. The advantages of the application of parallel FL to the ANS problem can be summarized as follows.

(i) The expected huge complexity involved in the heterogeneous environment is greatly reduced using the idea of parallel FLC that reduce the number of needed inference rules and the complexity of each rule. We have avoided the exponential increase of the rule base, and thus the increase in the complexity of the decision, when the number of input variables increases. To illustrate this point assume that we have two RATs only, five inputs per RAT to one FL system and every input has 3 membership functions, then we will have up to $3^{10}$ inference rules. In our scheme, if we use five parallel FLC with the same inputs and number of networks, we will have $5 * 3^{2}$ rules only.

(ii) The idea of the parallel FLC reduces the complexity of the inference rules used in the fuzzy-based solutions. To illustrate this point assume that we have five inputs per RAT to one fuzzy control system in HWN that have two RATs. The most complex inference rule will be as follows if $\mathrm{c} 1 / \mathrm{N} 1$ AND $\mathrm{c} 1 / \mathrm{N} 2$ AND $\mathrm{C} 1 / \mathrm{N} 3$ AND $\mathrm{c} 1 / \mathrm{N} 4$ AND c1/N5 AND c2/N1 AND c2/n2 AND $\mathrm{c} 2 / \mathrm{N} 3$ AND $\mathrm{c} 2 / \mathrm{N} 4$ AND c2/N 5 AND c3/n 1 AND c3/n2 AND c3/N3 AND c3/n 4 AND c3/n5 AND $\mathrm{c} 4 / \mathrm{N} 1$ AND $\mathrm{c} 4 / \mathrm{N} 2$ AND $\mathrm{c} 4 / \mathrm{N} 3$ AND $\mathrm{c} 4 / \mathrm{N} 4$ AND c4/N5 AND c5/N1 AND c5/N2 AND c5/n3 AND c5/N4 AND c5/N5 THEN D1 AND D2 AND D3 AND D4 AND D5, where ci/n $j$ means network, $j$ the rating value for criteria $i$, and $\mathrm{D} j$ means initial score for network $j$. However, using our parallel FLC, the most complex inference rule will be as follows If $\mathrm{C} 1 / \mathrm{N} 1$ AND $\mathrm{C} 1 / \mathrm{N} 2$ AND $\mathrm{C} 1 / \mathrm{N} 3$ AND $\mathrm{c} 1 / \mathrm{N} 4$ AND C1/N5 Then D1 AND D2 AND D3 AND D4 AND D5.

(iii) The output of the parallel FLC are in the range [0, 1] provide already normalize inputs (i.e., RATs rating against criteria) for the MCDM. This provides great advantage over the current MCDM-based solution that need to normalize their input data with some inappropriate methods.

\subsection{The Advantages of the Application of MCDM to the ANS Problem}

The framework utilizes the advantages of the application of MCDM to the ANS problem that can be summarized as follows.

(i) In a very complex and uncertain environments such as HWN, MCDM can sufficiently reduce the uncertainty and doubt about the alternatives and allows a reasonable choice to be made from among them.

(ii) ANS problem is a multicriteria problem in nature and the flexibility and complementary ANS multicriteria have to be utilized to provide a solution that can cope with the different view points and goals. If suitable weights have been assigned for each criterion using proper optimization methods such as GA, the user satisfaction average and the operator benefit can be maximized at the same time. This makes the proposed solution more efficient than the traditional algorithms that are either user centric or operator centric. 


\subsection{The Advantages of the Application of GA to the ANS Problem}

In our study, the GA is used to overcome one of the MCDM drawbacks, which is the finding of suitable weights for the different criteria. The following points summarize the main advantages of applying the GA to the ANS problem.

(i) GA can deal with the large number of variables and the complex search space included on the ANS criteria weights with high probability of success in finding optimal, near optimal, or at least good solution.

(ii) GA can handle the different constraints and objectives of the ANS criteria weights.

(iii) GA does not require derivative information, which can help out in the user-centric algorithm where not enough data is existed due to the limitation of storage in the mobile terminal.

(iv) GA is less likely to be trapped by local optimal minima or maxima.

(v) GA works with numerically generated data, experimental data, or analytical functions. This can give different options when designing the ANS algorithm.

\section{ANS SA for Coexisted CDMA- and TDMA-based Systems}

User software assistant (USA) based on our scheme with suitable input criteria can be used in the user equipment. Operator software assistant (OSA) based on this scheme with suitable input criteria can be used in the operator CRRM or JRRM entity. Based on the scheme proposed on the last section, ANS software assistants for users and operators can be developed. Since both OSA and USA are based on the same scheme and the main difference will be in the used input criteria, a general SA is presented in this section. The proposed SA deals with a wireless environment that contains a CDMA-based and a TDMA-based wireless networks. The details of the general SA is shown in Figure 2. The components of the SA can be summarized in Sections 3.1, 3.2, 3.3.

\subsection{The FL-based Control Component}

Our SA contains four FL-based subsystems. Each subsystem considers one of the ANS important criteria. The RSS subsystem considers the received signal strength criteria. The MSS subsystem considers the mobile station speed criteria. The ST subsystem considers the service-type criteria. The UP subsystem considers the user preference and price criteria. RSS subsystem has two input variables RSS1 to describe the received signal strength from CDMA-based network and RSS2 to describe the received signal strength from TDMAbased network. MSS subsystem has only one input variable MSS to describe the mobile station speed. ST subsystem has two input variables, the first is "DelayReqc" to describe the one-way delay needed for the required service and the second is "RateReqc" to describe the bit rate needed for the required service. UP subsystem has only one input variable "Price" to describe the user preferred price. Every input variable has three membership functions \{low, medium, high . Figure 3 shows the membership functions of the input variables.

Every subsystem has two output variables, one variable to describe the probability of acceptance for the new user in the CDMA network and the other variable to describe the probability of acceptance for the new user in the TDMA network. Each output variable has four membership functions $\{$ TR (totally reject), PR (probability reject), PA (probability accept), and TA (totally accept)\}. The subsystems output variables are "RSS $c 1$ " and "RSS ${ }_{c 2}$ " for RSS subsystem, "MSS ${ }_{c 1}$ " and "MSS $c 2$ " for MSS subsystem, " $\mathrm{ST}_{c 1}$ " and "ST $\mathrm{T}_{c 2}$ " for ST subsystem, and " $\mathrm{UP}_{c 1}$ " and " $\mathrm{UP}_{c 2}$ " for UP subsystem. Figure 4 shows " $\mathrm{ST}_{c 1}$ " variable with its membership functions as a sample for the output variables.

\subsection{The MCDM Component}

Enhanced version of simple multiattribute rating technique (SMART) has been used. SMART is one of the simplest MCDM and most efficient methods. The ranking value $x_{j}$ of alternative $A_{j}$ is obtained simply as the weighted algebraic mean of the utility values associated with it, that is, $a_{i j}$ according to (1):

$$
x_{j}=\frac{\sum_{i=1}^{m} w_{i} a_{i j}}{\sum_{i=1}^{m} w_{i}}, \quad j=1,2, \ldots, n .
$$

SMART employs relatively uncomplicated and straightforward manipulation method, which makes it stronger and easier to use in a hybrid and more complex models such as the proposed one in this paper. With the aid of both FL and GA, SMART has all the capabilities required to address the specific considerations that are involved in the ANS decision making process. SMART can be quickly and easily understood by the inexperienced decision makers. However, to get better understanding and deeper insights into the selection decision making, other advanced MCDM methods can be used in the future work and compared with SMART method.

In our algorithm, there are two alternatives for the MCDM, one is a CDMA-based network and the other is a TDMA-based network. The input criteria of the MCDM are the outputs of the FL-based control subsystems in the first component. The GA component assigns the weight $w_{i}$ for criteria $i$ to reflect its relative importance. The criteria with more importance to the operator and user can be assigned higher weight using the objective function of the GA specified by the operator. Since all the outputs of FL subsystems are in the range $[0,1]$, there is not be any need to scale the criteria performance against alternatives.

The ranking value of CDMA network $x_{c}$ and the ranking value of TDMA network $x_{t}$ can be calculated as follows: 


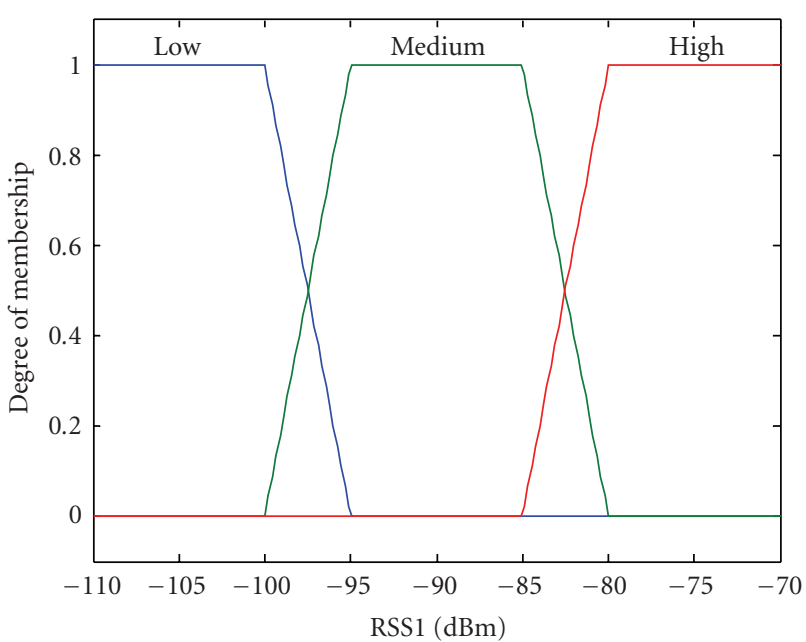

(a)

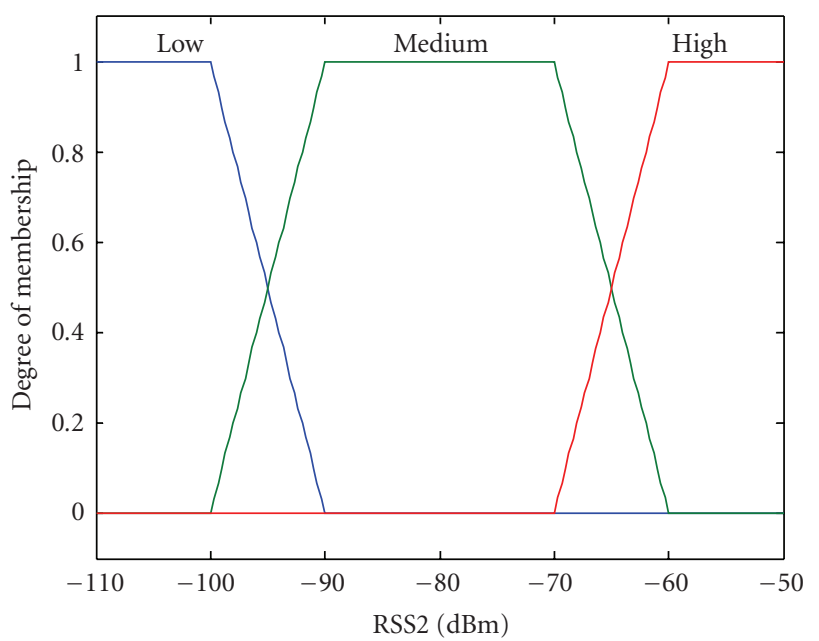

(c)

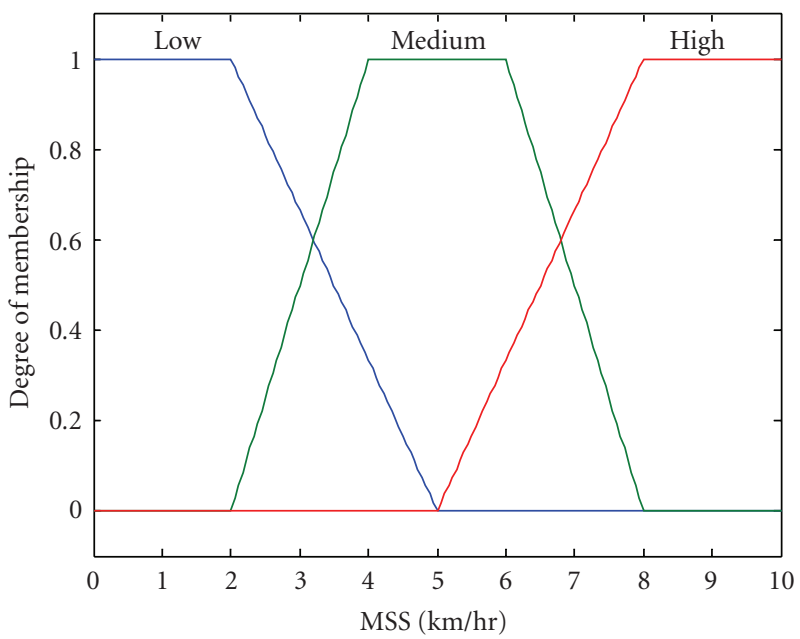

(e)

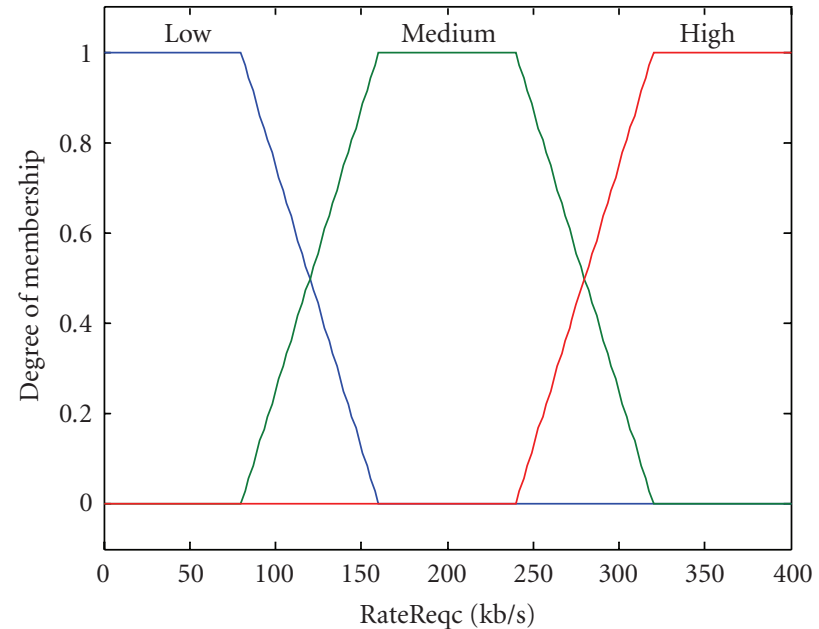

(b)

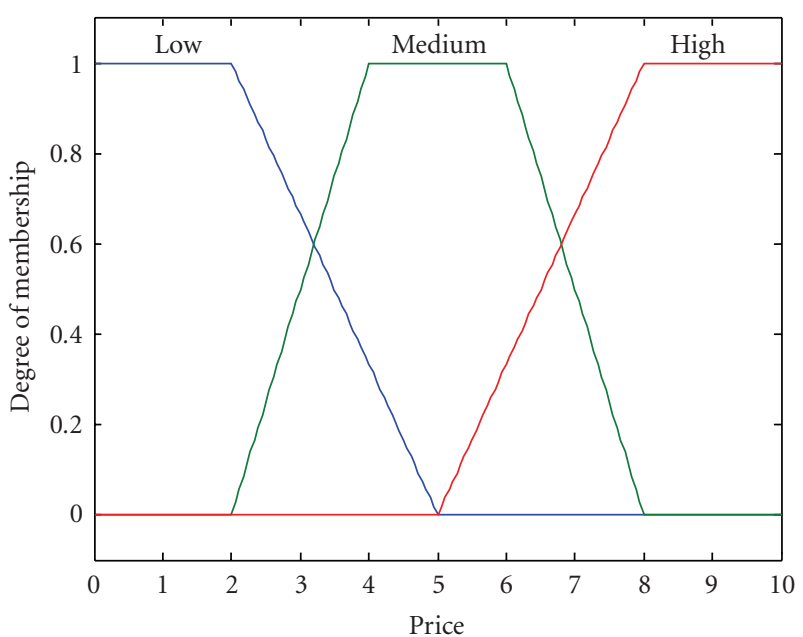

(d)

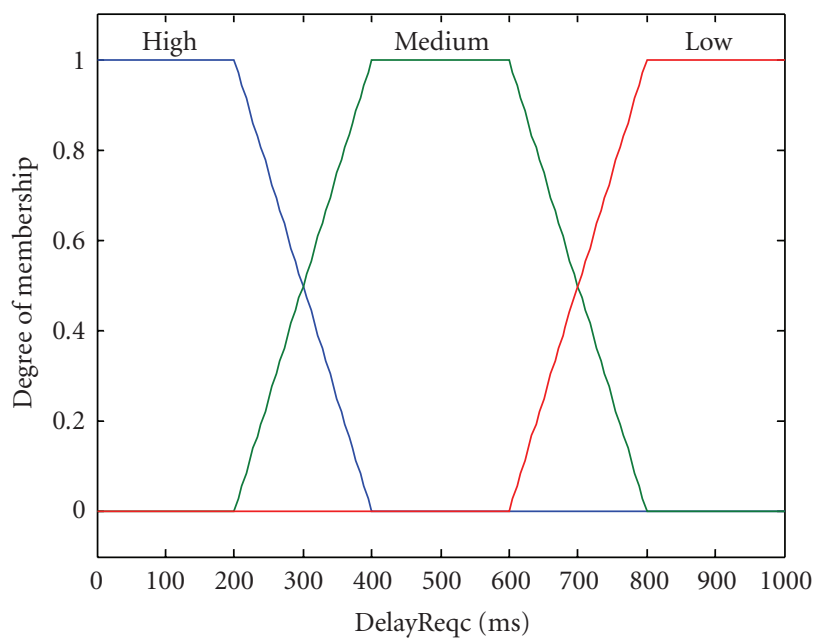

(f)

FIGURE 3: Membership functions of the input variables. 


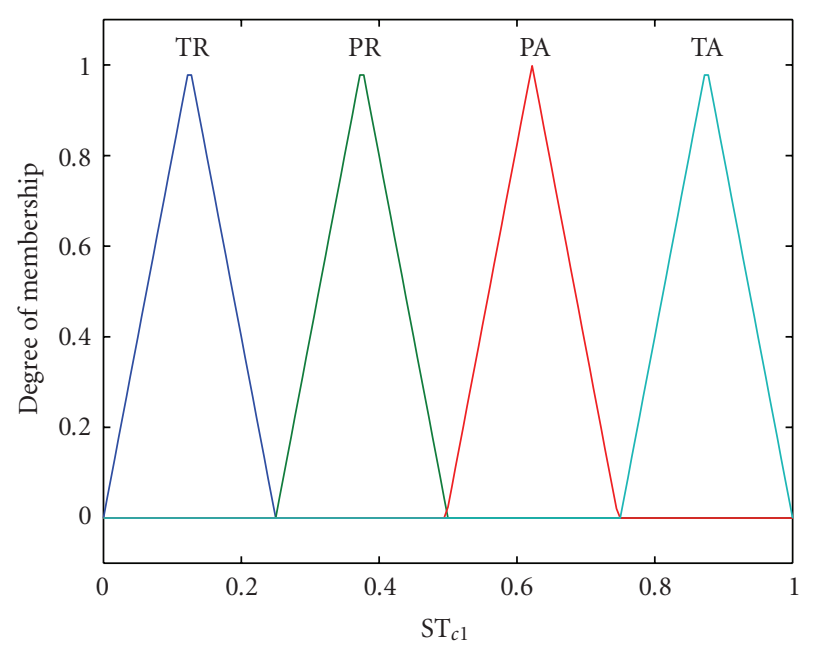

FIGURE 4: Membership functions for " $\mathrm{ST}_{c 1}$ " output variable.

$$
\begin{aligned}
& x_{c}=\frac{\mathrm{MSS}_{c 1} * W_{v}+\mathrm{RSS}_{c 1} * W_{s}+\mathrm{ST}_{c 1} * W_{t}+\mathrm{UP}_{c 1} * W_{u}}{T W}, \\
& x_{t}=\frac{\mathrm{MSS}_{c 2} * W_{v}+\mathrm{RSS}_{c 2} * W_{s}+\mathrm{ST}_{c 2} * W_{t}+\mathrm{UP}_{c 2} * W_{u}}{T W},
\end{aligned}
$$

where $W_{v}$ is the assigned weight for the mobile station speed criteria. $W_{s}$ is the assigned weight for the received signal strength criteria. $W_{t}$ is the assigned weight for the service type criteria. $W_{u}$ is the assigned weight for the user preferred price criteria. $T W$ is the total weight and is calculated using (3):

$$
T W=W_{v}+W_{s}+W_{t}+W_{u} .
$$

\subsection{The GA Component}

The GA has been used in our scheme to help the users or network operator to find suitable values for the weights $\left\{W_{v}, W_{s}, W_{t}, W_{u}\right\}$ in the offline mode. Beside the GA advantages that are summarized on Section 2.4, our decision to use GA was based on the nature of our objective functions that have several dynamic and stochastic components, where any other derivative-based optimization method cannot perform well. Another important issue that encouraged the selection of GA to our problem is the high interaction between different variables. At the end, usually the GA is not used to find an optimal solution and most of the time it is considered as a very promising option to find good and acceptable solution. It is worthy to mention that GA is not used on every selection decision. It is optional component on the scheme where the user can use it or not in offline mode prior to the selection process.

Since the ANS is a multicriteria problem in nature, different objectives need to be optimized. In this paper, three different objective functions are proposed to cover the different and opposite objectives and requirements of the users, QoS and operators. The proposed objective functions are outlined in the following points.

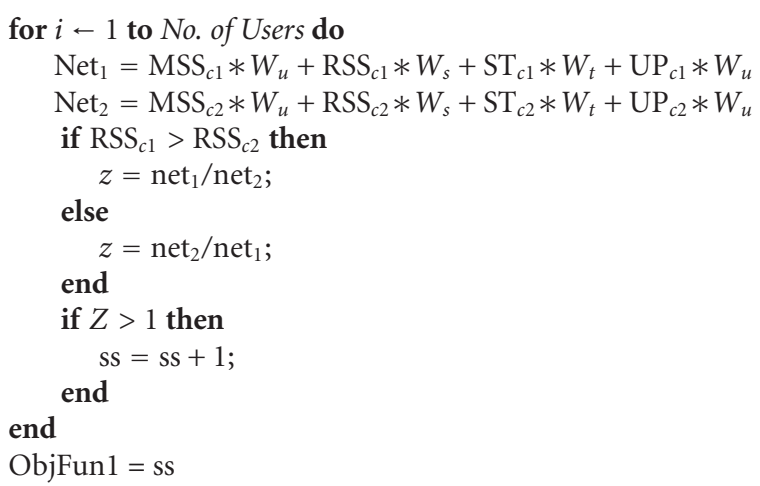

Algorithm 1: Pesudeocode for objective function ObjFun1.

(i) The first objective is to maximize the percentage of satisfied users, that is, users who are assigned to a network of their preference $\left(P_{u}\right)$ : since the algorithm considers multiple criteria, it may assign a user to a network different from his/her original preference. $P_{u}$ is considered appropriate for performance evaluation from the user point of view. To maximize $P_{u}$, the objective function ObjFun1 shown in Algorithm 1 is used.

(ii) The second objective is to maximize the percentage of users assigned to the networks with stronger signal strength $\left(P_{q}\right)$ : since the algorithm considers multiple criteria, it may assign a user to a network with weaker signal strength. $P_{q}$ is considered as a simple indicator for performance evaluation from the QoS point of view. To maximize $P_{q}$, the objective function ObjFun2 shown in Algorithm 2 is used.

(iii) The third objective is to achieve the load balancing between networks: $P_{o}$ is the percentage between the number of users in both networks. It is used as a basic indicator for load balancing. If the resources of both networks have the same importance and cost for network operator, this objective is considered appropriate for performance evaluation from network operator point of view. To achieve load balancing, the objective function ObjFun3 shown in Algorithm 3 is used.

The weights of the input criteria $\left\{W_{v}, W_{s}, W_{t}, W_{u}\right\}$ have been encoded using real encoding method. The length of the real-valued encoding is 4 real floating numbers. The length of each floating number is depending on the internal precision and roundoff used by the computer to define the precision of the floating numbers.

The results achieved by extensive comparisons of GA performance as a function of the different GA parameters (i.e., the population size, the mutation rate, and the crossover fraction) and the different GA operators (i.e., the selection and crossover operators) have been summarized by $\mathrm{R}$. L. Haupt and S. E. Haupt [27]. They have concluded that crossover fraction, selection operator, and crossover 


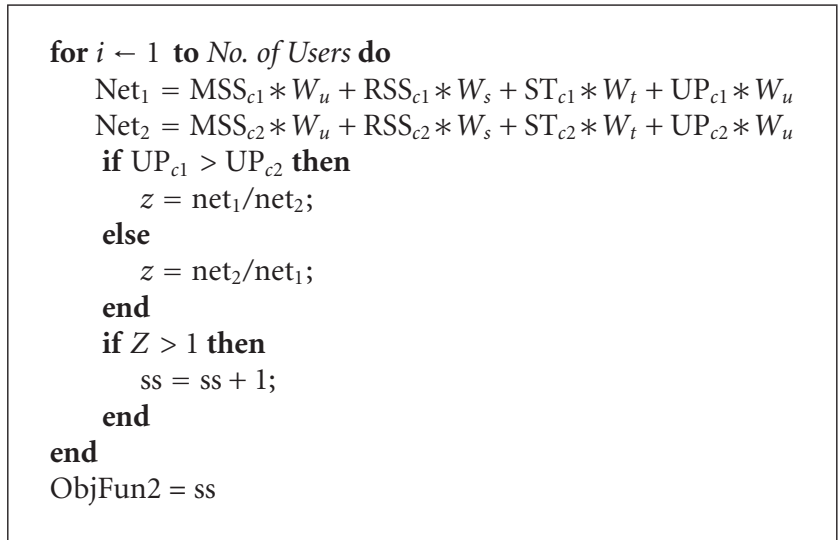

Algorithm 2: Pesudeocode for objective function ObjFun2.

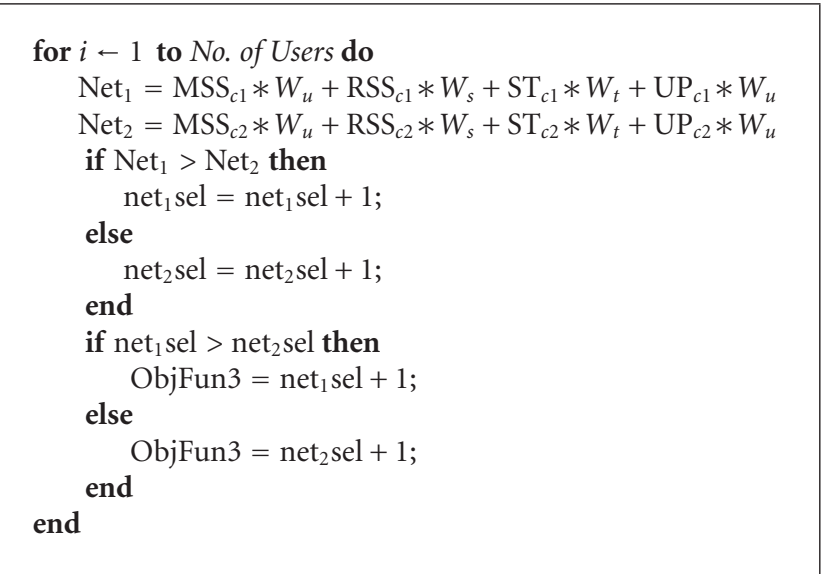

Algorithm 3: Pesudeocode for objective function ObjFun3.

operator are not of much importance. On the other side, the population size and the mutation rate have the most significant impact on the ability of the GA to find better minimum value for objective function.

Based on that, several sets of experiments to determine good population size and mutation rate for our proposed objective functions have been carried out. Each experiment has been repeated several iterations while the performance is recorded. The results from all the iterations are then combined by calculating the average (and standard deviation) for each experiment. Usually, testing GAs includes mainly two issues; how far the result obtained by GA is from the benchmark results, which can be measured by average fitness value. The second issue is how fast GA is in finding the best solution, which can be measured by the total number of function evaluations. Since our GA is working offline, only the average best fitness value achieved by the GA is used. The average best fitness and the standard deviation are plotted against the tested parameters. When studying a parameter all the other parameters and operators are kept constant.

Only a sample for the experiments results achieved is given in this paper as shown in Figure 5. The lower plot in Figure 5 shows the means and standard deviations of

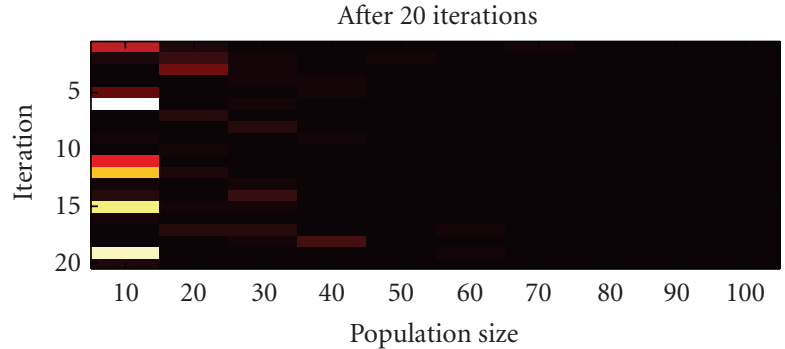

(a)

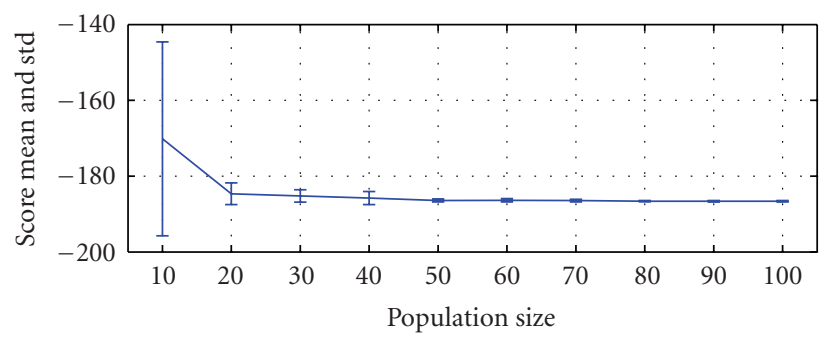

(b)

Figure 5: The effect of population size on objective function ObjFun1.

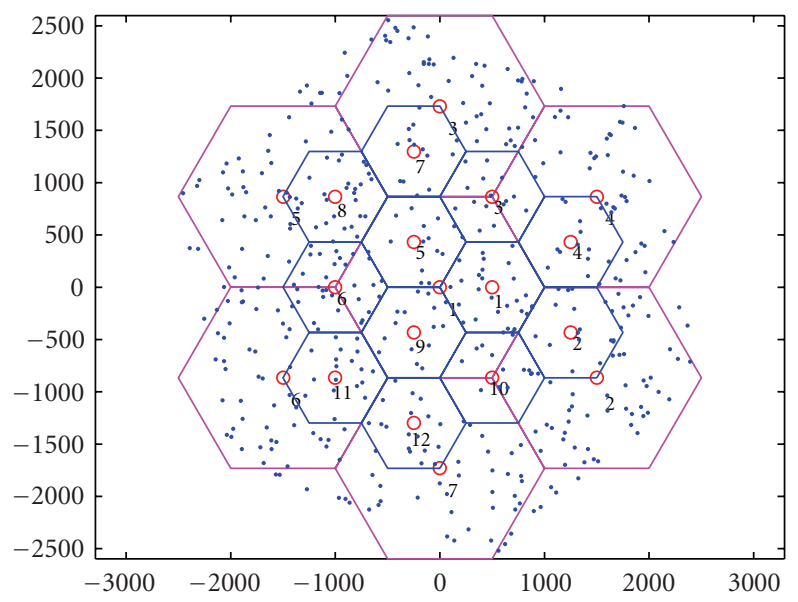

Figure 6: The users over the simulated environment.

the ObjFun1 function best fitness values over 20 runs, for each of the values of the population sizes between 10 and 100 with step size equals to 10 individuals. The upper plot shows a color-coded display of the best fitness values in each run where dark color indicates better results. For ObjFun 1 fitness function, setting the population size to 20 individuals or more yields better result. Based on these results and the other experiments results for other objective functions, the population size chosen in our GAs has been set to 20 . Other sets of experiments have been carried out to determine a suitable mutation rate. Based on the experiments, the mutation rate has been set to 0.1 .

In addition, our GA uses a roulette selection function. To ensure elitism, the number of individuals that are guaranteed to survive to the next generation has been set to 4 individuals. It uses two-point crossover function and a uniform mutation 


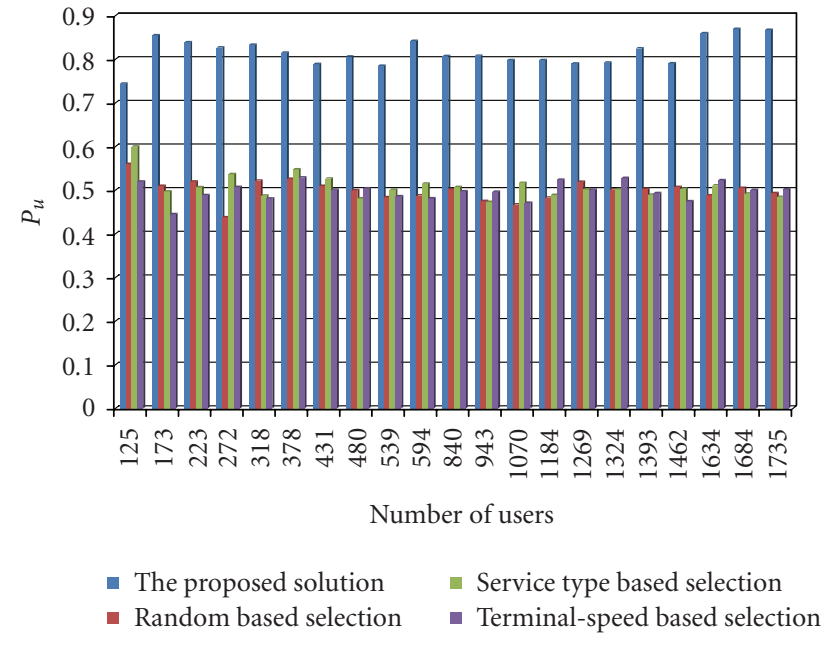

Figure 7: The percentage of satisfied users $\left(P_{u}\right)$ in the proposed algorithm against the three references algorithms.

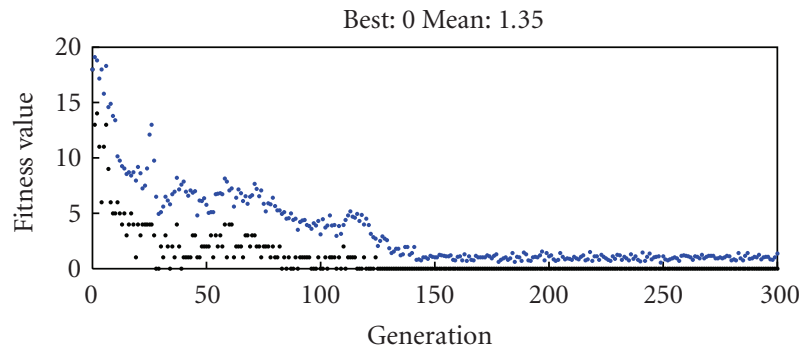

- Best fitness

- Mean fitness

(a)

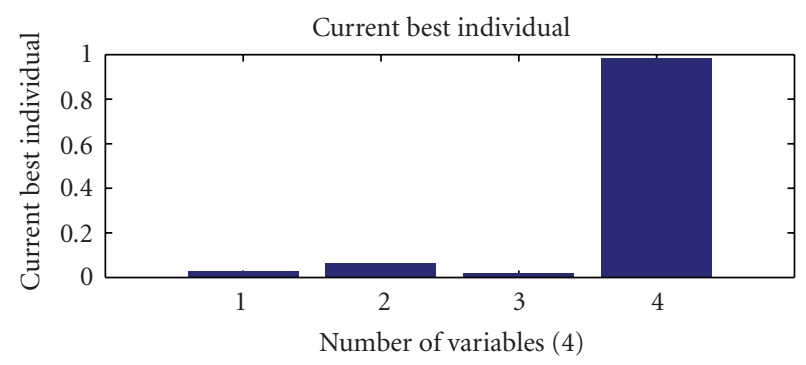

(b)

FIgURE 8: The weights assigned by GA for the 1st objective.

function. The GA terminates if the maximum number of the GA iterations reaches to 300 iterations, if there is no improvement in the best fitness value for number of consecutive generations equals to 100 generation, or if there is no improvement in the best fitness value for an interval of time equals to 300 seconds.

\section{The Performance Evaluation}

The used simulation models and some simulation results are presented in this section.

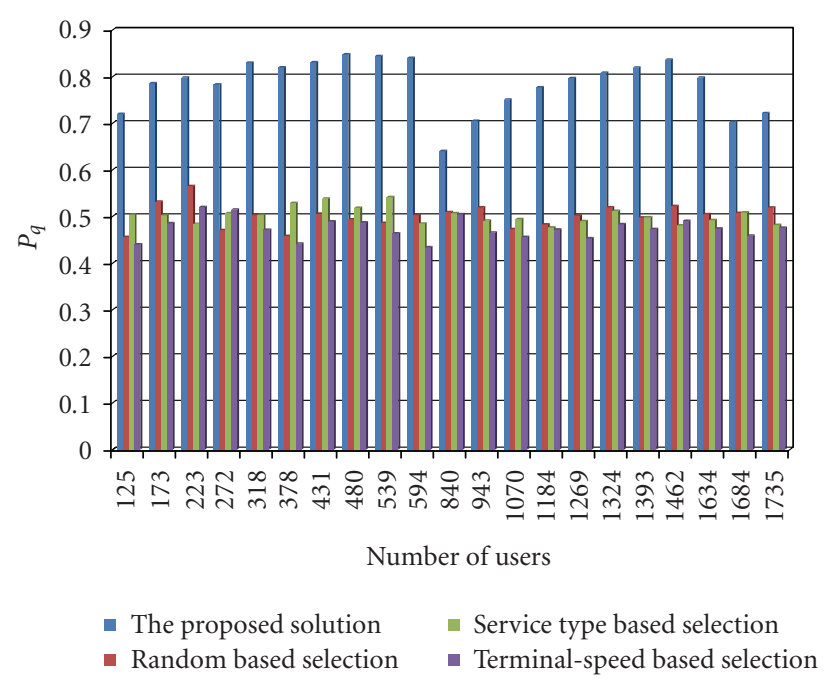

Figure 9: The percentage $P_{q}$ values in the proposed algorithm against the three references.

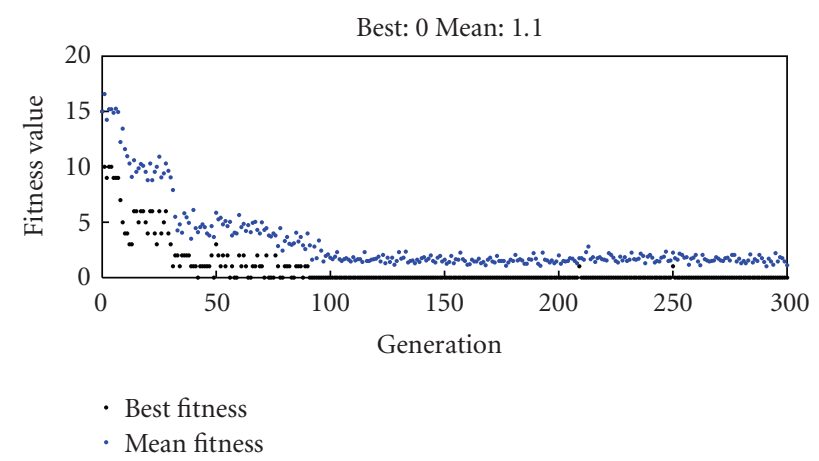

(a)

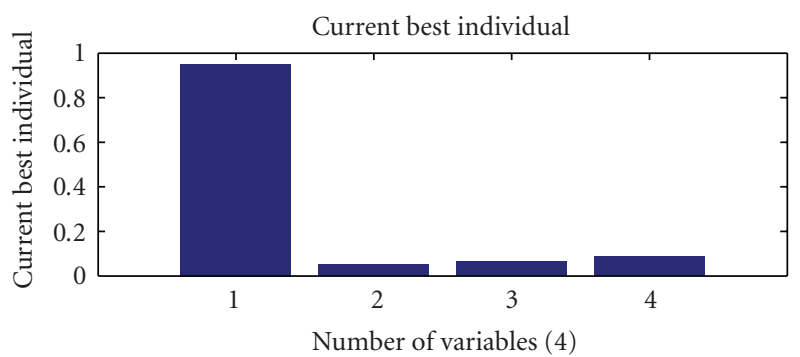

(b)

FIGURE 10: The weights assigned by GA for the 2nd objective.

\subsection{The Simulation Models}

Our proposed scheme and SA are evaluated using the simulation approach. MATLAB mathematical software and a set of functions called RUNE [1] have been used for the simulation. The system model considers the coexistence of a CDMA-based WWAN network with seven macrocells with omnidirectional antenna and cell radius $=1000 \mathrm{~m}$ and a TDMA-based WLAN network with twelve microcells with omnidirectional antenna and cell radius $=500 \mathrm{~m}$. In the system environment, each mobile has a velocity and is moved 
TABLe 1: $P_{u}$ values in all solutions.

\begin{tabular}{|c|c|c|c|c|}
\hline $\begin{array}{l}\text { Users } \\
\text { No. }\end{array}$ & $\begin{array}{c}\text { The proposed } \\
\text { solution } P_{u}\end{array}$ & $\begin{array}{c}\text { Random-based } \\
\text { selection } P_{u}\end{array}$ & $\begin{array}{c}\text { Service type-based } \\
\text { selection } P_{u}\end{array}$ & $\begin{array}{c}\text { Terminal speed-based } \\
\text { selection } P_{u}\end{array}$ \\
\hline 125 & 0.744 & 0.560 & 0.600 & 0.520 \\
\hline 223 & 0.839 & 0.520 & 0.507 & 0.489 \\
\hline 318 & 0.833 & 0.522 & 0.487 & 0.481 \\
\hline 431 & 0.789 & 0.510 & 0.527 & 0.501 \\
\hline 539 & 0.785 & 0.484 & 0.501 & 0.486 \\
\hline 840 & 0.807 & 0.502 & 0.507 & 0.498 \\
\hline 1070 & 0.798 & 0.467 & 0.517 & 0.471 \\
\hline 1269 & 0.790 & 0.519 & 0.502 & 0.501 \\
\hline 1393 & 0.825 & 0.503 & 0.490 & 0.493 \\
\hline 1634 & 0.860 & 0.488 & 0.511 & 0.523 \\
\hline 1735 & 0.867 & 0.493 & 0.485 & 0.502 \\
\hline
\end{tabular}

TABLe 2: $P_{q}$ values in all solutions.

\begin{tabular}{lcccc}
\hline $\begin{array}{l}\text { Users } \\
\text { No. }\end{array}$ & $\begin{array}{c}\text { The proposed } \\
\text { solution } P_{q}\end{array}$ & $\begin{array}{c}\text { Random-based } \\
\text { selection } P_{q}\end{array}$ & $\begin{array}{c}\text { Service-type-based } \\
\text { selection } P_{q}\end{array}$ & $\begin{array}{c}\text { Terminal speed-based } \\
\text { selection } P_{q}\end{array}$ \\
\hline 125 & 0.720 & 0.456 & 0.504 & 0.440 \\
223 & 0.798 & 0.565 & 0.484 & 0.520 \\
318 & 0.830 & 0.503 & 0.503 & 0.472 \\
431 & 0.831 & 0.506 & 0.538 & 0.490 \\
539 & 0.844 & 0.486 & 0.542 & 0.464 \\
840 & 0.640 & 0.510 & 0.507 & 0.505 \\
1070 & 0.750 & 0.473 & 0.494 & 0.456 \\
1269 & 0.797 & 0.502 & 0.490 & 0.453 \\
1393 & 0.819 & 0.497 & 0.498 & 0.473 \\
1634 & 0.598 & 0.505 & 0.492 & 0.474 \\
1735 & 0.622 & 0.519 & 0.482 & 0.476 \\
\hline
\end{tabular}

with a random distance and a random direction at defined time steps. The velocity is a vector quantity with magnitude and direction. The velocity of the $i$ th mobile is updated according to (4);

$$
U_{i}=U_{i} * P+\sqrt{1-P^{2}} * V_{m} * X
$$

where $U_{i}$ is the complex speed $[\mathrm{m} / \mathrm{s}] . P$ is the correlation of the velocity between time steps. $X$ is Rayleigh distributed magnitude with mean 1 and a random direction. $V_{m}$ is the mean speed of mobiles. Figure 6 shows the users, in blue, over the simulated environment. Four types of services are considered in our simulation, the voice calls, the low bit rate real-time video telephony, the high bit rate streaming video, and the nonreal-time data traffic. The traffic is modeled according to Poisson process. The main call holding time is assumed to be 50 seconds.

The propagation model used in this paper can be described in logarithmic scale as in (5)

$$
G=G_{D}+G_{F}+G_{R}+G_{A}
$$

Equation (5) contains four components. The first component is the distance attenuation $G_{D}$, which is given by the Okumura-Hata formula (6):

$$
G_{D}=-\{\beta+10 \alpha * \log (d)\},
$$

where $d$ is the distance to the transmitter, $\beta$ is a constant used to model the effect of carrier frequency, the antenna size and other physical parameters. The parameter $\alpha$ is the distance attenuation coefficient. $\beta$ has been set to $-28 \mathrm{~dB}$ and $\alpha$ has been set to 3.5 . The second component is the shadow fading $G_{F}$, which is modeled as a log-normal distribution with standard deviation of $6 \mathrm{~dB}$ and $0 \mathrm{~dB}$ mean. The third component is the Rayleigh fading $G_{R}$, which is a Rayleigh distributed. The fourth component is the antenna gain $G_{A}$.

\subsection{The Simulation Results}

This subsection shows some simulation results and compares the performance of our proposed solution to three different reference ANS algorithms. The first algorithm is a servicetype-based selection algorithm where high bit services with low propagation delay requirements are sent to the WLAN 
TABLE 3: $P_{o}$ values in all solutions.

\begin{tabular}{lcccc}
\hline $\begin{array}{l}\text { Users } \\
\text { No. }\end{array}$ & $\begin{array}{c}\text { The proposed } \\
\text { solution } P_{o}\end{array}$ & $\begin{array}{c}\text { Random-based } \\
\text { selection } P_{o}\end{array}$ & $\begin{array}{c}\text { Service type-based } \\
\text { selection } P_{o}\end{array}$ & $\begin{array}{c}\text { Terminal speed-based } \\
\text { selection } P_{o}\end{array}$ \\
\hline 125 & 1.341 & 1.155 & 0.984 & 0.944 \\
223 & 1.159 & 1.139 & 0.848 & 0.741 \\
318 & 1.196 & 0.989 & 0.935 & 0.866 \\
431 & 1.078 & 0.927 & 1.025 & 0.882 \\
539 & 1.355 & 0.968 & 0.900 & 0.855 \\
840 & 1.169 & 1.053 & 1.175 & 0.939 \\
1070 & 1.232 & 1.007 & 0.856 & 0.882 \\
1269 & 1.178 & 1.130 & 1.046 & 0.856 \\
1393 & 1.063 & 1.048 & 0.911 & 0.870 \\
1634 & 1.212 & 0.937 & 1.030 & 0.888 \\
1735 & 1.171 & 0.924 & 1.175 & 0.877 \\
\hline
\end{tabular}

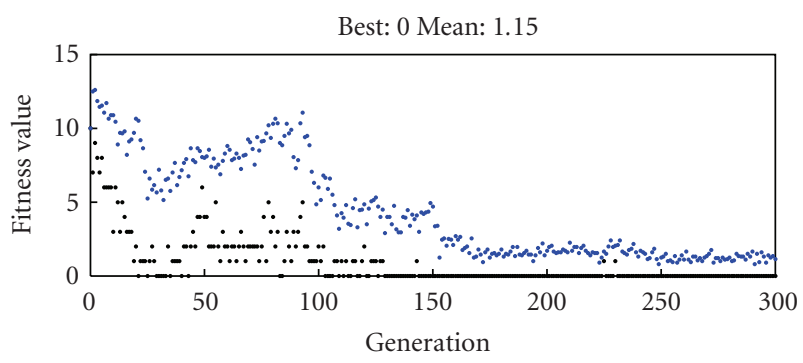

- Best fitness

- Mean fitness

(a)

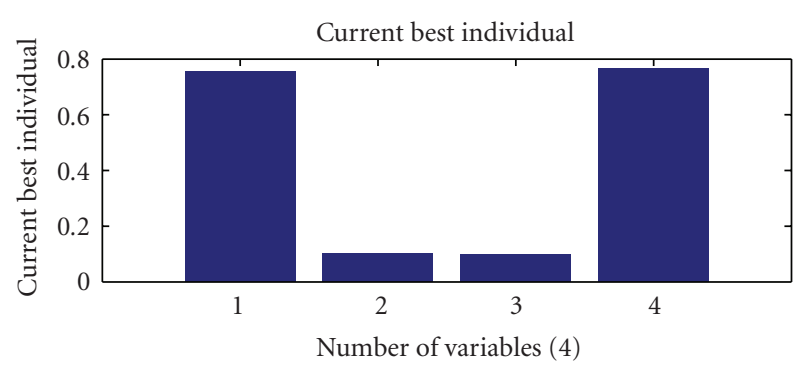

(b)

FIgURE 11: The weights assigned by GA for the 3rd objective.

and the low bit rate services with the high propagation delay requirements are sent to the WWAN. The second algorithm is a terminal speed-based selection algorithm where highspeed users are sent to the large-coverage network (i.e., WWAN) and the low-speed users are sent to the smallcoverage network (i.e., WLAN). The third algorithm is a random-based selection algorithm where the users are assigned randomly to the two networks. All solutions have been simulated, evaluated, and compared for the three different objectives addressed in Section 3.3. Several runs of simulation have been carried out. Each run uses a different number of users.

Figure 7 shows $P_{u}$ values in all solutions. The horizontal axis shows the number of users while the vertical axis shows the $P_{u}$ values. From both Figure 7 and the numerical samples for $P_{u}$ values shown in Table 1, the great improvement in the number of the satisfied users in our solution can be seen. For example, with 840 users in the environment, the percentage of satisfied users with all different reference solutions is around $50 \%$ while the same percentage in our proposed solution is around $80.7 \%$. In general, our proposed solution achieves around 31\% enhancement over the different reference algorithms. In Figure 8, the lower plot shows the weights assigned by the GA for the different criteria using the first objective function. The upper plot shows the best function value in each generation versus iteration number.

Figure 9 shows $P_{q}$ values in all solutions. The horizontal axis shows the number of users while the vertical axis shows the $P_{q}$ values. From both Figure 9 and the numerical samples for $P_{q}$ values shown in Table 2, the great improvement in the percentage of the users assigned to networks with stronger received signal can be seen. For example, with 840 users in the environment $P_{q}$ with the different reference algorithms is around $50 \%$ while the same percentage in our proposed solution is around $64 \%$. In average, our proposed solution achieves around 26.5\% enhancement over the random-based selection. Figure 10 shows the weights assigned by the GA for the second objective.

The results of $P_{o}$ produced by all solutions for the third objective are shown in Table 3. Our proposed solution achieves good and comparable results against the results achieved by other solutions. Figure 11 shows the weights assigned by the GA for the third objective.

\section{Conclusions}

A general scheme to solve the multicriteria ANS problem has been presented in this paper. The proposed scheme is scalable and is able to handle any number of RATs with a large set of criteria. The scheme can cope with the different and contrast view points and goals of the operator and users and it can react to the accumulated human knowledge about the problem. The proposed scheme has been used to present and design a multicriteria ANS solution that considered the 
user, the QoS, and the operator view points. The simulation results show that the proposed solution has a better and more robust performance over the reference solutions.

Different aspects of our work have to be developed. More criteria can be included such as resources availability and signal-to-noise ratio (SNR). Multiobjective optimization method can be used to find an optimum weights jointly rather than using the single-objective GA that optimize each objective weight independently. The used rules and membership functions of the fuzzy subsystems are still subjective, so they can be built or tuned using a suitable learning method such as the genetic algorithms or the neural networks. Our algorithm can be compared with more reference algorithms.

\section{References}

[1] J. Zander and S. Kim, Radio Resource Management for Wireless Networks, Artech House, Boston, Mass, USA, 2001.

[2] J. Pérez-Romero, O. Sallent, R. Agustí, and M. A. Díaz-Guerra, Radio Resource Management Strategies in UMTS, John Wiley \& Sons, New York, NY, USA, 2005.

[3] M. Cardei, I. Cardei, and D.-Z. Du, Resource Management in Wireless Networking, Springer, New York, NY, USA, 2005.

[4] 3GPP TR 25.881, "Improvement of RRM across RNS and RNS/BSS," (Release 5), v5.0.0.

[5] 3GPP TR 25.891, "Improvement of RRM across RNS and RNS/BSS," (Post Rel-5), v0.3.0.

[6] A. Tlli, P. Hakalin, and H. Holma, "Performance evaluation of common radio resource management (CRRM)," in Proceedings of the IEEE International Conference on Communications (ICC '02), vol. 5, pp. 3429-3433, New York, NY, USA, AprilMay 2002.

[7] IST, "Evolutionary Strategies for Radio Resource Management in Cellular Heterogeneous Networks," (EVEREST) Project, http://www.everest-ist.upc.es/.

[8] IST, "End to End Reconfigurability," (E2R) Project, http:// e2r.motlabs.com/.

[9] IST, "Wireless World Initiative New Radio," (WINNER) Project, http://www.ist-winner.org/.

[10] IST, "Smart user-centric communications environment," (SCOUT) Project, http://www.ist-scout.org/.

[11] IST, "Transparently Reconfigurable Ubiquitous Terminal," (TRUST) Project, http://www.ist-trust.org/.

[12] Y. Gwon, D. Funato, and A. Takeshita, "Adaptive approach for locally optimized IP handoffs across heterogeneous wireless networks," in Proceedings of the 4th International Workshop on Mobile and Wireless Communications Network (MWCN '02), pp. 475-479, Stockholm, Sweden, September 2002.

[13] M. Ylianttila, M. Pande, J. Mäkelä, and P. Mähönen, "Optimization scheme for mobile users performing vertical handoffs between IEEE 802.11 and GPRS/EDGE networks," in Proceedings of the IEEE Global Telecommunications Conference (GLOBECOM '01), vol. 6, pp. 3439-3443, San Antonio, Tex, USA, November 2001.

[14] H. S. Park, S. H. Yoon, T. H. Kim, J. S. Park, M. S. Do, and J. Y. Lee, "Vertical handoff procedure and algorithm between IEEE802.11 WLAN and CDMA cellular network," in Proceedings of the 7th CDMA International Conference on Mobile Communications (CIC '02), pp. 103-112, Seoul, Korea, October-November 2002.
[15] W. Song, H. Jiang, W. Zhuang, and X. Shen, "Resource management for QoS support in cellular/WLAN interworking," IEEE Network, vol. 19, no. 5, pp. 12-18, 2005.

[16] X. Gelabert, J. Pérez-Romero, O. Sallent, R. Agustí, and F. Casadevall, "Radio resource management in heterogeneous networks," in Proceedings of the 3rd International Working Conference on Performance Modelling and Evaluation of Heterogeneous Networks ( HET-NETs'05), Ilkley, UK, July 2005.

[17] K. Murray and D. Pesch, "Policy based access management and handover control in heterogeneous wireless networks," in Proceedings of the 60th IEEE Vehicular Technology Conference (VTC '04), vol. 5, pp. 3319-3323, Los Angeles, Calif, USA, September 2004.

[18] J. Ylitalo, T. Jokikyyny, T. Kauppinen, A. J. Tuominen, and J. Laine, "Dynamic network interface selection in mobile hosts," in Proceedings of the 36th Annual Hawaii International Conference on System Sciences (HICSS '03), pp. 315-324, Big Island, Hawaii, USA, January 2003.

[19] F. Bari and V. Leung, "Application of ELECTRE to network selection in a hetereogeneous wireless network environment," in Proceedings of the IEEE Wireless Communications and Networking Conference (WCNC'07), pp. 3810-3815, Kowloon, Hong Kong, March 2007.

[20] F. Bari and V. Leung, "Multi-attribute network selection by iterative TOPSIS for heterogeneous wireless access," in Proceedings of the 4th IEEE Consumer Communications and Networking Conference (CCNC '07), pp. 808-812, Las Vegas, Nev, USA, January 2007.

[21] T. Al-Gizawi, K. Peppas, D. I. Axiotis, E. N. Protonotarios, and F. Lazarakis, "Interoperability criteria, mechanisms, and evaluation of system performance for transparently interoperating WLAN and UMTS-HSDPA networks," IEEE Network, vol. 19, no. 4, pp. 66-72, 2005.

[22] R. Pintenet, J. Gosteau, T. Al-Gizawi, F. Lazarakis, K. Peppas, and A. Alexiou, "Evaluation of interoperability mechanisms for coexisting HSDPA and WLAN enhanced with MTMR techniques," in Proceedings of the 58th IEEE Vehicular Technology Conference (VTC '03), vol. 3, pp. 1807-1811, Orlando, Fla, USA, October 2003.

[23] L. Liang, H. Wang, and P. Zhang, "Net utility-based network selection scheme in CDMA cellular/WLAN integrated networks," in Proceedings of the IEEE Wireless Communications and Networking Conference (WCNC '07), pp. 3313-3317, Kowloon, Hong Kong, March 2007.

[24] L. Giupponi, R. Agustí, J. Pérez-Romero, and O. Sallent, "A novel joint radio resource management approach with reinforcement learning mechanisms," in Proceedings of the 24th IEEE International Performance, Computing, and Communications Conference (IPCCC '05), pp. 621-626, Phoenix, Ariz, USA, April 2005.

[25] R. Agustí, O. Sallent, J. Pérez-Romero, and L. Giupponi, "A fuzzy neural based approach for joint radio resource management in a B3G framework," in Proceedings of the 1st International Conference on Quality of Service in Heterogeneous Wired/Wireless Networks (QSHINE '04), pp. 216-224, Dallas, Tex, USA, October 2004.

[26] A. Wilson, A. Lenaghan, and R. Malyan, "Optimizing wireless access network selection to maintain QoS in heterogeneous wireless environments," in Proceedings of the Wireless Personal Multimedia Communications (WPMC '05), Aalborg, Denmark, September 2005.

[27] R. L. Haupt and S. E. Haupt, Practical Genetic Algorithms, John Wiley \& Sons, New York, NY, USA, 2nd edition, 2004. 

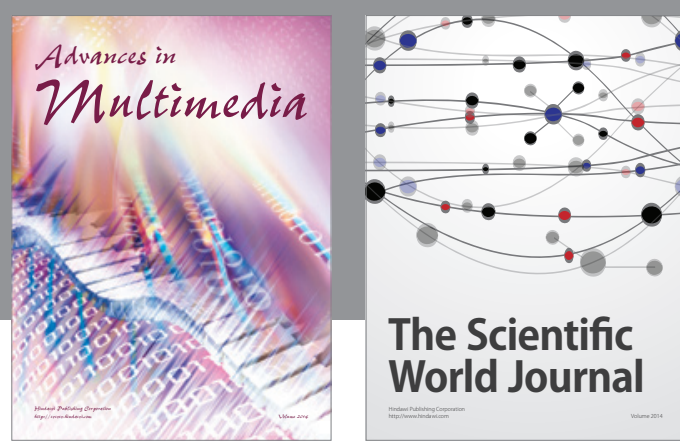

The Scientific World Journal
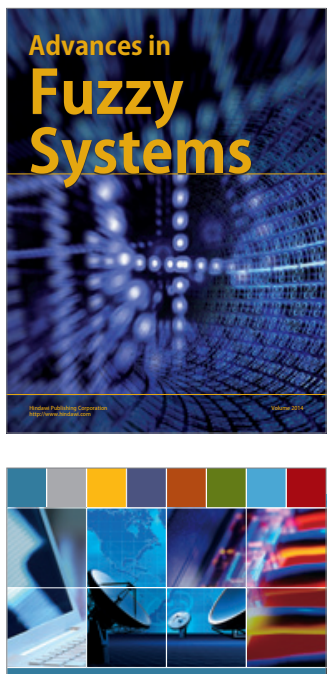

Computer Networks and Communications
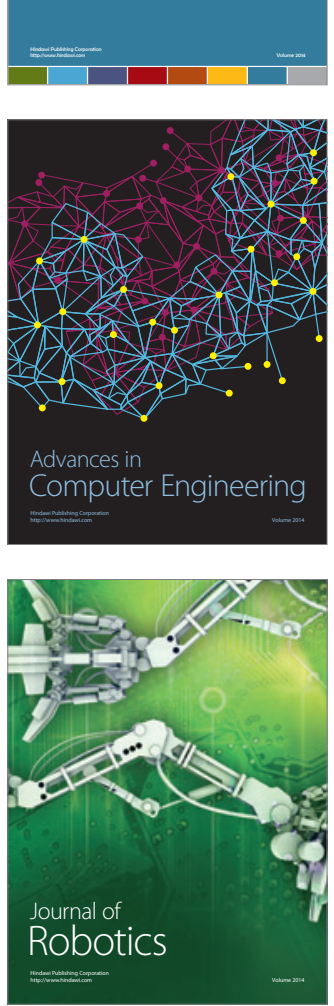
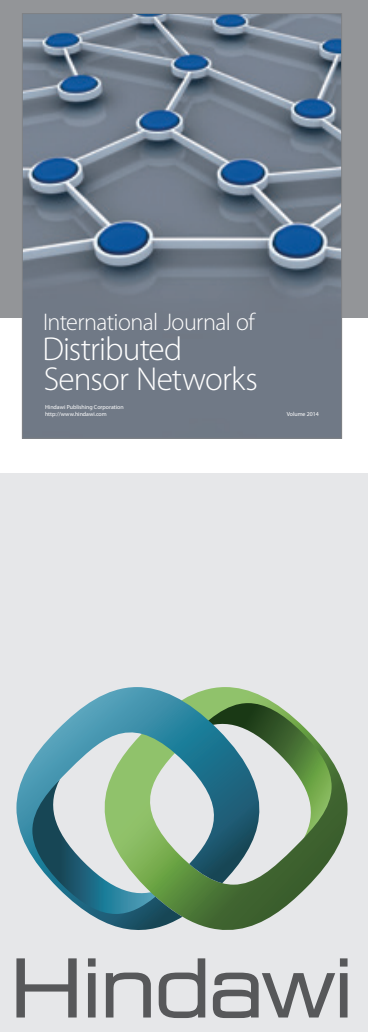

Submit your manuscripts at

http://www.hindawi.com
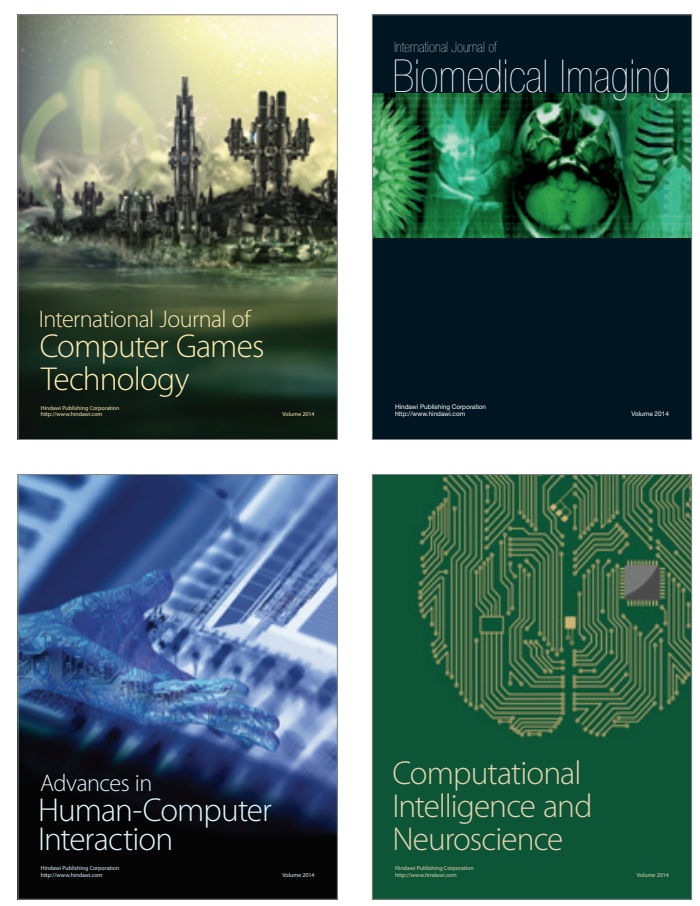
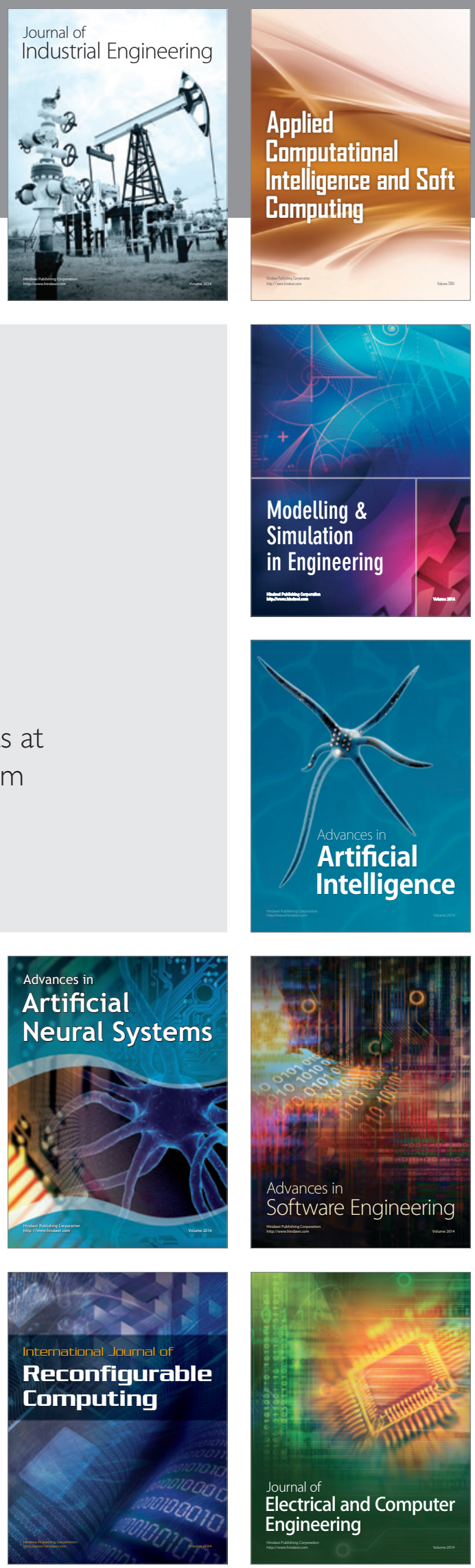